\title{
Arzneimittelsicherheit in klinischen Studien
}

Russmann, S

Posted at the Zurich Open Repository and Archive, University of Zurich ZORA URL: https://doi.org/10.5167/uzh-69950

Book Section

Accepted Version

Originally published at:

Russmann, S (2012). Arzneimittelsicherheit in klinischen Studien. In: Kullak-Ublick, G A; Siepmann, T; Kirch, W. Arzneimitteltherapie: Wirksamkeit - Sicherheit - Praktische Anwendung. Stuttgart: Thieme, 35-38. 


\subsubsection{Arzneimittelsicherheit in klinischen Studien}

\section{Stefan Russmann}

Die Grundlagen zur Sicherheit klinischer Studien sind in den Prinzipien der Good Clinical Practice (GCP) Richtlinien festgeschrieben. Deren Umsetzung wird insbesondere durch die zuständigen Ethikkommissionen und Arzneimittelbehörden überwacht, denen alle klinischen Studien in denen Medizinprodukte angewendet werden zur Prüfung vorgelegt werden müssen. Auch die GCP Richtlinien bauen auf dem eingangs vorgestellten Prinzip der RisikoNutzen Abwägung auf, und eine klinische Studie sollte demnach nur begonnen und weitergeführt werden, wenn der erwartete Nutzen das Risiko rechtfertigt. Somit müssen also bereits vor Beginn einer klinischen Studie umfangreiche sicherheitsrelevante Informationen aus präklinischen Versuchen und gegebenenfalls klinische Erfahrungen aus der Anwendung vergleichbarer Substanzen dokumentiert und bewertet werden. Risiken sollten dabei sowohl für das Individuum, als auch für die Gesellschaft gegen den erwarteten Nutzen gewichtet werden. Gleichzeitig wird in den GCP Richtlinien aber auch klargestellt, dass die Sicherheit und Rechte der Studienteilnehmer die höchste Priorität haben und über die Interessen von Gesellschaft und Wissenschaft gestellt sind. Versuchsteilnehmer müssen demnach über zu erwartende Risiken aufgeklärt werden und dies in der Einverständniserklärung schriftlich bestätigen. Auch die Studienärzte müssen in der sogenannten Investigator's Brochure über alle relevanten Sicherheitsaspekte und insbesondere über erwartete UAW informiert werden. Sowohl die jeweils verantwortlichen Studienärzte als auch die Patienten selbst können jederzeit unabhängig einen Studienabbruch einzelner Patienten aus Sicherheitsgründen veranlassen.

Die Erfassung und fortlaufende Beurteilung der Sicherheit einer klinischen Studie einschliesslich Erstellung von Zwischenberichten ist bereits im Studienprotokoll festzulegen und unterliegt a priori der Verantwortung des Studiensponsors. Das Studienprotokoll muss dementsprechend spezifische Informationen zu Sicherheitsparametern enthalten, welche vor und während der Studie zu bestimmen sind. Diese können zum Beispiel die wiederholte Messung von Leberwerten, Nierenfunktion, Blutbild, EKG und vollumfängliche klinische Untersuchungen beinhalten. Weiterhin müssen auch Methoden und Zeitplan für die Evaluation der erhobenen Sicherheitsparameter sowie von unerwünschten Ereignissen 
inklusive Art und Dauer von deren geplanter Nachverfolgung im Protokoll beschrieben sein. Zudem sollte ein in seiner Beurteilung vom Sponsor unabhängiges Gremium zur Sicherheitsüberwachung eingerichtet werden (englisch: Safety Monitoring Board). Neue sich während der Durchführung ergebende sicherheitsrelevante Informationen müssen zeitnah an die Ethikkommission, die Arzneimittelbehörden, das Safety Monitoring Board und an alle betroffenen Untersucher weitergeleitet werden. Insbesondere bei multizentrischen Studien ist dies wichtig, damit alle Untersucher fortlaufend über Sicherheitsprobleme, welche eventuell in anderen Zentren der Studie aufgetreten sind oder aus anderen Quellen stammen, informiert werden.

Für die Erfassung von unerwünschten Ereignissen gilt in klinischen Studien eine standardisierte Nomenklatur. Eine unerwünschte Arzneimittelwirkung (englisch: adverse drug reaction, ADR) meint in Zulassungsstudien alle schädlichen und unerwünschten Ereignisse, bei denen ein kausaler Bezug mit einem Medizinprodukt zumindest möglich erscheint. Ein unerwünschtes Ereignis (englisch: adverse event, AE) im Rahmen einer klinischen Studie impliziert hingegen lediglich einen zeitlichen Zusammenhang zwischen einem unerwünschten medizinischen Ereignis und der Studienmedikation, wohingegen die Frage nach dem kausalen Zusammenhang hier explizit ausgeklammert wird. Die Unterscheidung zwischen Adverse events und Adverse drug reactions erscheint auf den ersten Blick umständlich, die Erfassung aller unerwünschten Ereignisse ist aber notwendig, damit auch UAW, welche zunächst nicht als solche erkannt wurden, erfasst und dokumentiert werden. Bei allen unerwünschten Ereignissen erfolgt die erste Beurteilung eines eventuellen kausalen Zusammenhangs mit der Studienmedikation durch den Studienarzt. Zusätzlich nimmt der Sponsor anschließend eine eigene Beurteilung vor, da diese aber von Interessenskonflikten des Sponsors beeinflusst sein könnte, muss auch die ursprüngliche Beurteilung durch den Untersucher original dokumentiert und an die zuständigen Arzneimittelbehörden weitergeleitet werden. Die Kausalitätsbeurteilung von UAW in klinischen Studien erfolgt ähnlich den im Kapitel Pharmakovigilanz beschriebenen Methoden durch Zuordnung zu einer Kausalitätskategorie, genaue Vorgaben hierzu bestehen aber nicht. Der Schweregrad wird ebenfalls beurteilt, wobei schwerwiegende Ereignisse (englisch: serious) definitionsgemäß solche sind, welche zum Tode führen, lebensbedrohlich sind, eine Hospitalisierung bedingen oder eine solche verlängern, irreversible Schäden zur Folge haben, sowie kindliche Missbildungen.

Klinische Studien eignen sich aufgrund dieser klaren Vorgaben zur Erfassung und dementsprechend zuverlässigen Dokumentation aller unerwünschten Ereignisse a priori gut zur Quantifizierung von unerwünschten Ereignissen, und wegen der standardisierten 
Bedingungen und des Vorhandenseins einer randomisierten Kontrollgruppe auch zur Bestimmung des kausalen Zusammenhangs mit der Studienmedikation.

Gerade deshalb ist zu bemängeln, dass gemäß einer 2001 veröffentlichten umfangreichen Untersuchung auch wichtige Sicherheitsinformationen aus klinischen Studien in den entsprechenden Publikationen neben den dort in den Vordergrund gestellten Ergebnissen zur Wirksamkeit oft nur unzureichend enthalten sind.

Wie eingangs bereits dargestellt (siehe hierzu auch Tabelle 1 in der Einführung zu Kapitel 1.3) unterliegen klinische Studien bei der Beurteilung der Arzneimittelsicherheit aber auch wichtigen intrinsischen Limitationen. Vor allem ist das Design klinischer Zulassungsstudien auf den Wirksamkeitsnachweis hin optimiert. Schon die Fallzahlberechnung richtet sich nicht nach ausreichender statistischer Power für die Quantifizierung von seltenen UAW. So wäre gemäß der „Rule of three“ für eine UAW mit einer Häufigkeit von 1:1000 der Einschluss von mindestens 3000 exponierten Patienten in eine Studie erforderlich. Weiterhin haben viele Studien eine kurze Dauer, und in diesem Fall können UAWs mit langer Latenzzeit gar nicht erst nicht erfasst werden. Auch entsprechen die Bedingungen unter denen klinische Studien durchgeführt werden kaum denen im klinischen Alltag. Die Compliance bezüglich regelmäßiger Medikamenteneinnahme in der richtigen Dosis ist in klinischen Studien gewöhnlich überdurchschnittlich gut, und Gruppen mit erhöhter Prävalenz von Risikofaktoren für UAW, welche die Medikamente dann nach Marktzulassung aber oft dennoch verschrieben bekommen, werden evtl. systematisch ausgeschlossen. Dies betrifft insbesondere ältere Patienten mit weiteren Begleiterkrankungen und dementsprechend oft zusätzlicher Medikamenteneinnahme mit Interaktionspotential. Immerhin sollten Zulassungsstudien heute Risikogruppen doch zumindest so gut wie möglich berücksichtigen, speziell Patienten mit eingeschränkter Nieren- und Leberfunktion. Und auch eine ausgeglichene Verteilung bezüglich Alter, Geschlecht und ethisch-genetischer Faktoren wird angestrebt.

Informationen zu Art und Häufigkeit von in klinischen Studien aufgetretenen unerwünschten Ereignissen werden schließlich in der Fachinformation und der Patienteninformation eines Medikaments dokumentiert. Häufigkeitsangaben können gemäß internationaler Vorgaben dabei nur absolut, oder zusätzlich im Vergleich zu Kontrollgruppen gemacht werden, wobei letzteres aber informativer ist und daher wenn immer möglich angestrebt werden sollte. Insgesamt spielt die Arzneimittelsicherheit auch in klinischen Studien eine zunehmend wichtigere Rolle und wird dementsprechend auch immer besser erfasst und ausgewertet. Seltene Nebenwirkungen und die Sicherheit eines Medikamentes im klinischen Alltag 
können in klinischen Studien aber nicht ausreichend beurteilt werden, weshalb es zusätzlich der Pharmakovigilanz und der Pharmakoepidemiologie bedarf. 